\title{
Two-column two-filter water intake well and the method of its calculation
}

\author{
V.V. Ivashechkin, J.A. Medvedeva, E.S. Satsuta \\ Belarusian National Technical University, Department of Hydraulic and power \\ engineering, water transport and hydraulics, Belarus, Minsk
}

\begin{abstract}
Boreholes are the most versatile and technically advanced structural type of water intake structures. In this article, the authors propose the design of a water intake well with filters located at two levels, capable of simultaneously performing the functions of a working and reserve wells, the use of which will reduce energy consumption for lifting water, reduce capital investments for construction and ensure uninterrupted water supply. The methodology for calculating the inflow of water, constructing hydrodynamic grids, determining the function of lowering the pressure and filtering features during the operation of a two-level well in a confined aquifer is also presented.
\end{abstract}

\section{INTRODUCTION}

Boreholes are the most versatile and technically advanced structural type of water intake structures. Currently, in the Republic of Belarus and most European countries, groundwater is extracted using water wells. The extraction of groundwater for the water supply of large settlements is carried out using high-yield water wells. In Belarus, the average service lifetime of high-yield water wells of standard designs is 20-23 years [1,2].

With intensive exploitation of wells in most cases, their productivity decreases due to the chemical and biological origin sedimentation of filters in holes, gravel pores, and aquifers. A direct consequence of colmatage is an increase in filtration rates, an increase in input hydraulic resistances, and a decrease in water flow to the well. Despite the application of measures to regenerate filters, sedimentations accumulate and are not removed during treatments. A failed well is plugged back and a new one is built instead, which is not only irrational and expensive but also unfavorable from an environmental point of view, as corrosive metal structures remain in the exploited underground horizon. This indicates the insufficient efficiency of 
the existing methods of regeneration and the fragility of the existing typical well designs. To increase the reliability and durability of groundwater intake and ensure uninterrupted water supply to the consumer, it is often envisaged to install two water intake wells: the main and the reserve ones, which operate alternately and therefore have an increased service life. However, the costs of the construction of two wells are doubled, is the zone of sanitary protection, where the special regime of operation conditions of the territory protection are organized. This arrangement of the placement of the working and reserve wells involves significant material costs for the construction of individual wells with pavilions and networks, and also requires the alienation of significant areas under the territory of their sanitary protection zones. To intensify the water intake, when required to obtain the best performance at a given point of the aquifer, two-column wells developed by V. P. Tkachenko can be installed. In a two-column well, an above-filter pipe is used as the first drive tube, and a bypass pipe is cut into a settling basin and installed parallel to the filter in the gravel package circuit. In this case, the pumps are mounted in a bypass pipe and drive tube with a depth below the minimum level of 1-3 $\mathrm{m}$ (according to operating conditions). The operation of the well with two pumps installed in two columns allows the alignment of the velocity profile on the filter. However, during a major workover of such a well, it is impossible to remove and replace the filter. Therefore, in the case of a significant decrease in the productivity of a well, when current repairs are ineffective, such a well is plugged back and a new one is built instead, which narrows the scope of its application $[3,4,5]$.

Therefore, the purpose of the work was to develop a design for a twocolumn water intake well, combining the reliability and durability of the working and reserve wells, as well as the methodology for its hydraulic calculation.

BNTU proposed the design of a two-column two-filter water intake well (Fig. 1). The well consists of a conduit with annular cementation, the first borehole consisting of a production string, a filter with a working section and a settling basin, the second borehole consisting of a production string, a filter with a working section, and a settling basin, an upper connecting pipe, a lower connecting pipe, gravel package, sand backfill, clay key, injection tubes with perforation, made opposite the working section of the filters. 


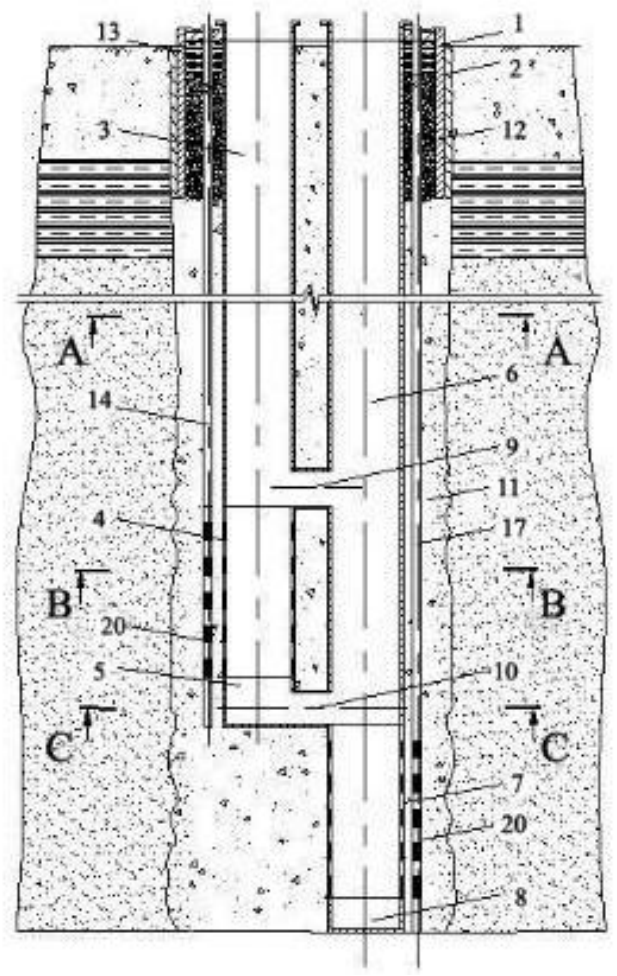

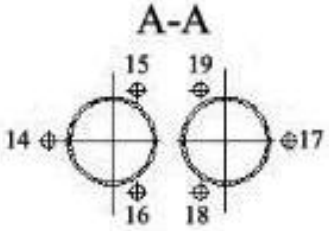

B-B
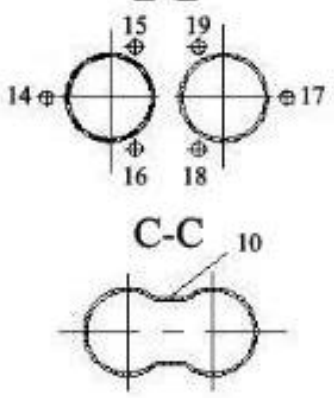

Fig. 1. Construction of a two-column two-level water well, 1 - conduit; 2 - annular cementation; 3 - production string of the first borehole; 4 - filter of the first borehole with a working section; 5 - settling basin of the first borehole; 6 production string of the second borehole; 7 - filter of the second borehole with a working section; 8 - settling basin of the second borehole; 9 - upper connecting pipe; 10 - lower connecting pipe; 11 - gravel package; 12 - sand backfill; 13 - clay key; 14-19 - injection tubes; 20 - perforation;

The well can be constructed as follows: the sediment bulb is dug out and the exploration well is drilled with a drill bit to the target depth. Geophysical surveys are carried out in the exploration borehole to determine the depth and thickness of the aquifer. After decoding the well logs, the guide string is installed, the borehole is drilled under the conductor by the cable drilling method, the annular cementation of the conductor is performed to its entire height up to the mouth of the well. The curing time for conductors is usually set at 24 hours. Then, lowering the drill bit through the conductor to the bottom of the well, the cement plug in the conductor is drilled out, the boreholes drilled opening the aquifer to the required depth. Since drilling is carried out with a large diameter, it is performed by the method of backwashing with clean water. On the ground, at the mouth of the well, the lower connecting pipe is welded with one end into the settling basin and the other end into the hole of the lower part of the production string. Then the upper connecting pipe is welded with one end into the hole in the first 
production string, and the other end into the hole of the second production string. The resulting welded structure is suspended on the crane boom, joined and welded to a filter, and then lowered to the bottom of the well, building up production strings by welding. To ensure a uniform layer of gravel package around the filters, they are equipped with special centralizers. In the process of lowering, injection pipes are fixed to the centralizers, with perforations made opposite the filters. The top of the injection pipes is brought out to the mouth of the well. After that, gravel is backfilled into the space between the injection pipes and the first and second boreholes to create a gravel package around the filters. The gravel stock above the top of the filters should be at least $5 \mathrm{~m}$. Airlifts are lowered into the first and second boreholes and the development of the aquifer is carried out by pulsating pumping, which can be combined with swabbing. If necessary, gravel can be filled up when pumping. Then unwashed sand is poured on top of the gravel to the mouth of the well. The well is equipped with a head, and two boreholes are equipped with separate downhole pumps.

\subsection{The operation of a two-level water intake well can be carried out as follows}

When water is taken from a two-column well with one pump located in the second borehole, the main flow is fed through the filter of the second borehole, and the flow rate coming from the filter of the first borehole through the upper connecting pipe is added to it. Both filters work.

When water is taken from a two-column well with two pumps located, respectively, in the first and second wells, both filters also work. Each filter has its own filtration flow from the water-bearing stratum. As the filters are located at different levels of the aquifer column, the fluid flows are separated and they do not significantly affect each other. This Will allow the filtration rate on the way to the filters to be reduced.

If it is necessary to regenerate the filters and their near-filter zones, circulating reagent washing is performed. Submersible pumps from both boreholes are dismantled. A special container with a reagent is installed at the wellhead. The tank is equipped with filling hoses for supplying the reagent and airlift for pumping out dissolution products. When regenerating the filter through the filling hoses, the reagent is first fed by gravity into three injection pipes located at an angle of $120^{\circ}$ to each other. The reagent enters through the perforation of the injection pipes into the filter zone near the filter. At the same time, dissolution products are pumped out of the filter of the first borehole, installed here by airlift, into a container with a reagent. Airlift creates a depression inside the filter and ensures the continuous circulation of the reagent in the filter and gravel package. This ensures the required intensity of dissolution of deposits and the quality of processing. For example, hydrochloric acid or an acidic cleaning agent "Descam" can be used as a reagent for dissolving deposits.

The advantage of the two-level well is that the filters are installed in a common water receiver, not opposite each other but at different levels, which 
increases water intake capacity. Two radial seepage flows are formed along the height of the reservoir when this installation of filters works with the simultaneous operation of two pumps and with the operation of any of the pumps. It will allow the filtration rate on the way to the filters to be reduced. This will create conditions for reducing pressure losses and will decline of a water table in the well, providing a reduction in the cost of produced water, increase in specific yield of a well and its lifetime in comparison with a single-column design. Also, the proposed design is more reliable, because if there is a hydraulic connection between the two columns in case of failure of one filter, working with one pump or two at the same time is possible.

The disadvantages of the proposed design include the fact that for the upper filter, since it is located in the gravel package near the production string of the lower filter, some distortion of streamlines may be observed.

When describing the operation of a two-column two-level water intake well in an aquifer, dependencies were used to determine the decrease in pressure and build hydrodynamic filtration flow nets of water inflow to the well. The proposed design was reduced to the design scheme shown in Figure 2 , where it is assumed that the well filters have a common vertical axis.

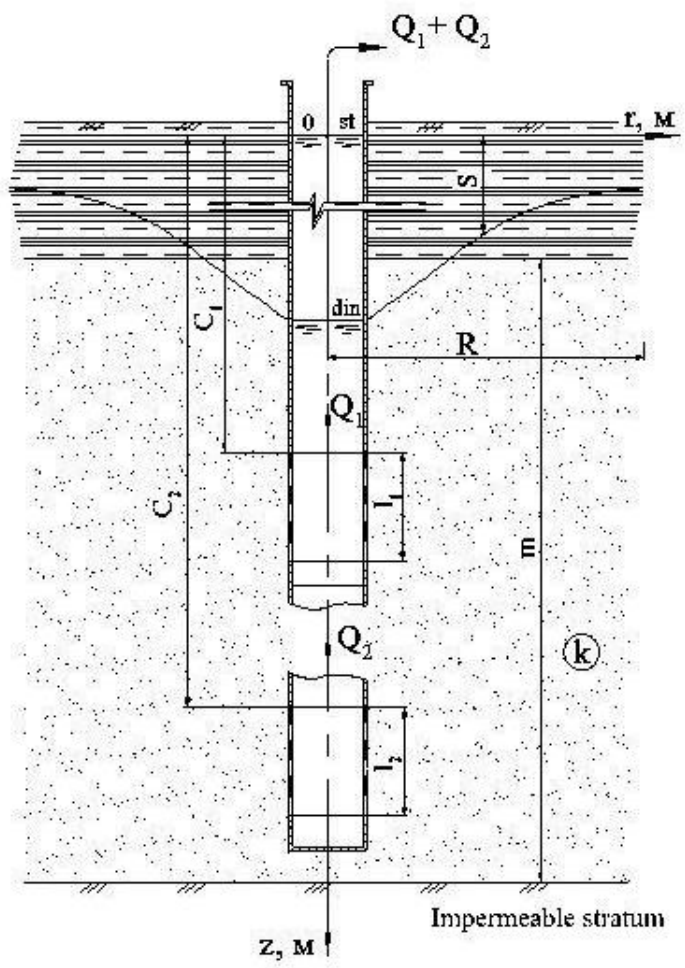

Fig. 2. The design scheme of a two-level well.

We accept a confined aquifer underlain by relatively less permeable rocks. Consider the work of a two-level well in anaquiferous stratum 
underlain by relatively less permeable rocks. Using the dependence of finding the function of decreasing the pressure during the work of a two-level well with filters, obtained by adding the flows to two wells, it is possible to determine the pressure losses at any point in the radius of the influence of pumping:

$$
s=s_{1}+s_{2}
$$

where $s_{1}=$ pressure losses obtained during the operation of the upper filter; $s_{2}=$ pressure losses obtained when the lower filter is operating.

$$
\begin{gathered}
s_{1}=\frac{Q_{1}}{4 \pi k}\left[f \frac{c_{1}+\dot{l}_{1}-z}{r}+f \frac{c_{1}+\dot{l}_{1}+z}{r}-f \frac{2 m-c_{1}-\dot{l}_{1}-z}{r}+\frac{l_{1}}{m}\left(2 \ln \frac{R}{r}-\right.\right. \\
\left.\left.\quad \operatorname{arsh} \frac{2 m-z}{r}-\operatorname{arsh} \frac{m+z}{r}\right)\right], \\
S_{2}=\frac{Q_{2}}{4 \pi k}\left[f \frac{c_{2}+l_{2}-z}{r}+f \frac{c_{2}+\dot{l}_{2}+z}{r}-f \frac{2 m-c_{2}-\dot{l}_{2}-z}{r}+\frac{l_{2}}{m}\left(2 \ln \frac{R}{r}-\right.\right. \\
\left.\left.\operatorname{arsh} \frac{2 m-z}{r}-\operatorname{arsh} \frac{m+z}{r}\right)\right],
\end{gathered}
$$

where $Q_{1} \& Q_{2}=$ yield of upper and lower wells; $\mathrm{k}=$ aquifer filtration coefficient; $m=$ aquifer thickness; $l_{1}$ и $l_{2}=$ filter length of the upper and lower wells; $\lambda=Q_{1} / Q_{2}=$ yield ratio; $R=$ pumping radius; $r, z=$ cylindrical coordinates of a point, in which the pressure value is determined; $c_{1}=$ the distance of the upper end of the filter of the upper well from the edge of the aquifer; $c_{2}=$ same for the lower well filter.

The dot over $i$ in function $f$ means that the argument of the subtracted function $\operatorname{arsh}$ is not included in $l$, i. e.:

Function

Similarly, we have

$$
f \frac{c+l \pm z}{r}=\operatorname{arsh} \frac{c+l \pm z}{r}-\operatorname{arsh} \frac{c \pm z}{r}
$$

$$
f \frac{2 m-c-i \pm z}{r}=\operatorname{arsh} \frac{2 m-c-l \pm z}{r}-\operatorname{arch} \frac{2 m-c \pm z}{r} .
$$

Let us introduce the following notations:

$$
\begin{gathered}
A_{1}=f \frac{c_{1}+\dot{l}_{1}-z}{r}+f \frac{c_{1}+\dot{l}_{1}+z}{r}-f \frac{2 m-c_{1}-\dot{l}_{1}-z}{r}, \\
A_{2}=f \frac{c_{2}+\dot{l}_{2}-z}{r}+f \frac{c_{2}+\dot{l}_{2}+z}{r}-f \frac{2 m-c_{2}-\dot{l}_{2}-z}{r} .
\end{gathered}
$$

Substituting formulas (6) and (7), respectively, in (2) and (3), we get:

$$
\begin{aligned}
& s_{1}=\frac{Q_{1}}{4 \pi k}\left[A_{1}+\frac{l_{1}}{m}\left(2 \ln \frac{R}{r}-\operatorname{arsh} \frac{2 m-z}{r}-\operatorname{arsh} \frac{m+z}{r}\right)\right] \\
& s_{2}=\frac{Q_{2}}{4 \pi k}\left[A_{2}+\frac{l_{2}}{m}\left(2 \ln \frac{R}{r}-\operatorname{arsh} \frac{2 m-z}{r}-\operatorname{arsh} \frac{m+z}{r}\right)\right]
\end{aligned}
$$

Then, finally, the function of pressure losses at a given point will be obtained by adding the losses during the operation of the upper and lower filters.

$$
s=\frac{Q_{1}}{4 \pi k}\left[\frac{A_{1}}{l_{1}}+\frac{A_{2}}{\lambda l_{2}}-\frac{(1+\lambda)}{\lambda m}\left(\operatorname{arsh} \frac{2 m-z}{r}+\operatorname{arsh} \frac{m+z}{r}\right)+\frac{2(1+\lambda)}{\lambda m} \ln \frac{R}{r}\right] .
$$

To analyze the features of filtration with the following initial data given below, using dependence (10), we built hydrodynamic filtration flow nets to the well at various distances between the filters in height: 8 and 15 meters (Figure 3). 
For an example of the calculation, we will take the following initial data: $Q_{1}=Q_{2}=0,001 \mathrm{~m}^{3} / \mathrm{sec}, \quad k=0,0001 \mathrm{~m} / \mathrm{sec}, \quad m=22 \mathrm{~m}, \quad c_{1}=37 \mathrm{~m}$, $c_{2}=51$ (58) $m, l_{1}=l_{2}=6 \mathrm{~m}, R=200 \mathrm{~m}$ (filtration coefficient and radius of influence are taken according to reference data for medium-grained sands).
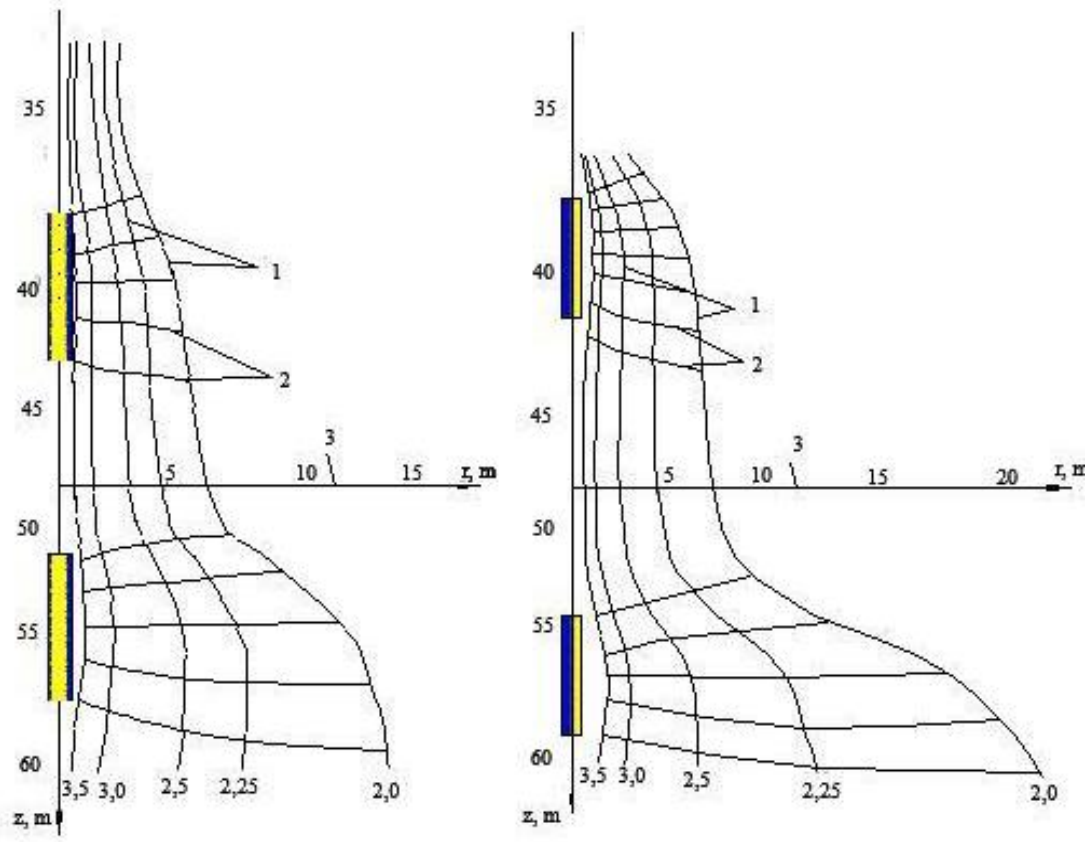

Fig. 3 - Hydrodynamic filtration flow nets

Here: $1=$ lines of equal pressure losses; $2=$ streamlines; $3=$ interface between streams. A) the distance between the filters is 8 meters; B) the distance between the filters is 15 meters.

$$
v_{0 z}=-\frac{Q_{1}}{4 \pi \Pi_{0}}\left[\frac{A_{1}^{\prime}}{l_{1}}+\frac{A_{2}^{\prime}}{\lambda l_{2}}+\frac{(1+\lambda)}{\lambda m}\left(\frac{1}{2 m-z}-\frac{1}{m+z}\right)\right] .(11
$$

Since the speed at this point should be zero, we get:

$$
\frac{A_{1}^{\prime}}{l_{1}}+\frac{A_{2}^{\prime}}{\lambda l_{2}}+\frac{(1+\lambda)}{\lambda m}\left(\frac{1}{2 m-z}-\frac{1}{m+z}\right)=0
$$

where $\Pi_{0}$ is the active porosity of an aquifer

$$
\begin{gathered}
A_{1}^{\prime}=\frac{1}{z-c_{1}}-\frac{1}{z-c_{1}-\dot{l}_{1}}+\frac{1}{z+c_{1}+\dot{l}_{1}}-\frac{1}{z+c_{1}}, \\
A_{2}^{\prime}=\frac{1}{c_{2}-z}-\frac{1}{c_{2}+\dot{l}_{2}-z}+\frac{1}{c_{2}+\dot{l}_{2}+z}-\frac{1}{c_{2}+z},
\end{gathered}
$$

For the case when the distance between the filters is 8 meters (Figure 3, A), the point of intersection of the flow interface with the z-axis was $48.2 \mathrm{~m}$, and for the case when the distance between the filters is 15 meters, it was $52.3 \mathrm{~m}$.

In the case when there is a watertight stratum, i.e., two filters operate in a homogeneous aquifer, pressure losses in the water level in the upper well, 
taking into account the additional pressure losses in the water level under the influence of the lower one, can be presented in the following form:

$$
s_{1}=\frac{Q_{1}}{2 \pi k m}\left(\ln \frac{R}{r_{0}}+\Phi_{\mathrm{H}}+\varphi_{2}\right)
$$

where $r_{0}=$ filter radius, $\Phi_{\mathrm{H}}=$ additional resistance to well imperfection, $\varphi_{2}=$ coefficient associated with the influence of the lower well and

$$
\Phi_{\mathrm{H}}=\frac{1-\bar{l}}{\bar{c}}\left(\ln \frac{l_{1}}{r_{0}}-\varepsilon\right),
$$

where $=f(\bar{b} ; \bar{l}) ; \bar{b}=m-b_{1} / m-l_{1} ; \bar{l}=l_{1} / m ; b_{1}=c_{1}+l_{1}$, i.e., the distance of the lower end of the upper filter from the top of the aquifer; $\bar{c}=c_{1}+l_{1} / c_{1}$.

Taking $z=b_{1}$, we represent the value of $\varphi_{2}$ in the following form:

$$
\varphi_{2}=\frac{1,15}{\lambda}\left(\frac{m}{l_{2}} \zeta_{2}+\log \frac{R^{2}}{\beta_{2}}\right) \text {, }
$$

where $\beta_{2}=\left(2 m-b_{1}\right)\left(m+b_{1}\right) \quad$ and $\quad \zeta_{2}=\log \left(b_{2}^{2}-b_{1}^{2} / c_{2}^{2}-b_{1}^{2}\right)+$ $\log \left(2 m-c_{2}-b_{1} / 2 m-b_{2}-b_{1}\right) ; b_{2}=c_{2}+l_{2}$ (see fig. 2).

Provided that the length of the filter of the lower well $l_{2}$ is small in comparison with the thickness of the aquifer, the second term in the expression $\zeta_{2}$ can be neglected, i.e.,

$$
\zeta_{2}=\log \frac{b_{2}^{2}-b_{1}^{2}}{c_{2}^{2}-b_{1}^{2}}
$$

Thus, the formula for the well yield of the upper well is expressed as follows:

$$
Q_{1}=\frac{2 \pi k m s_{1}}{2,3 \log \frac{R}{r_{0}}+\Phi_{\mathrm{H}}+\varphi_{2}} .
$$

The formula for calculating the well yield of the lower well or lowering the level in it can be obtained similarly:

$$
s_{2}=\frac{Q_{2}}{2 \pi k m}\left(2,3 \log \frac{R}{r_{0}}+\Phi_{\mathrm{H}}+\varphi_{1}\right)
$$

where $\Phi_{\mathrm{H}}$ is theadditional resistance to well imperfection,

$$
\varphi_{1}=1,15 \lambda\left(\frac{m}{l_{1}} \zeta_{1}+\log \frac{R^{2}}{\beta_{1}}\right),
$$

where $\beta_{1}=\left(2 m-c_{2}\right)\left(m+c_{2}\right) ; \zeta_{1}=\log \frac{c_{2}^{2}-b_{1}^{2}}{c_{2}^{2}-c_{1}^{2}}+\log \frac{2 m-c_{1}-c_{2}}{2 m-b_{1}-c_{1}}$.

If the filter length of the upper well $l_{1}$ is small compared to the thickness of the aquifer, then the second term in the expression for $\zeta_{1}$ can be neglected. Then: $\zeta_{1}=\log \left(c_{2}^{2}-b_{1}^{2} / c_{2}^{2}-c_{1}^{2}\right)$.

Using dependencies (10), (17), (20), the total pressure losses in a twolevel well are presented in Table 1.

Table 1.Determination of the pressure losses function

\begin{tabular}{cccccc}
\hline $\begin{array}{c}\mathrm{Q}, \\
\mathrm{m}^{3} / \mathrm{sec}\end{array}$ & $\begin{array}{c}\mathrm{k}, \mathrm{sec} \\
\mathrm{m} / 000\end{array}$ & $\begin{array}{c}\mathrm{l}_{1}=\mathrm{l}_{2}, \\
\mathrm{~m}\end{array}$ & $\mathrm{~m}, \mathrm{~m}$ & $\mathrm{R}, \mathrm{m}$ & $\mathrm{r}, \mathrm{m}$ \\
\hline 0.003 & $\begin{array}{c}0.000 \\
1\end{array}$ & 4.5 & 40 & 200 & 0.2 \\
\hline 0.003 & $\begin{array}{c}0.000 \\
1\end{array}$ & 4.5 & 45 & 200 & 0.2
\end{tabular}




\begin{tabular}{|c|c|c|c|c|c|}
\hline 0.003 & $\begin{array}{l}0.000 \\
1 \\
\end{array}$ & 4.5 & 50 & 200 & 0.2 \\
\hline $\mathrm{z}, \mathrm{m}$ & $\mathrm{c}_{1}, \mathrm{~m}$ & $\mathrm{c}_{2}, \mathrm{~m}$ & $\begin{array}{c}\mathrm{s} \\
(10), \mathrm{m} \\
\end{array}$ & s (17) & \\
\hline 31.5 & 25 & 33.5 & 3.2 & 2.5 & \\
\hline 32.5 & 25 & 37.5 & 2.5 & 2.2 & \\
\hline 34.5 & 25 & 44.5 & 2.15 & 1.98 & \\
\hline
\end{tabular}

The obtained close values of the pressure losses indicate the possibility of using the above dependencies (10), (17), (20) to describe the operation of a two-level well.

The presented method for calculating a two-column two-filter water intake well allows to determine the depressions at any point within the pumping influence radius, build hydrodynamic filtration flow nets, and also determine the most advantageous distance between the two filters in height to obtain a higher flow rate and uninterrupted operation of the proposed well design.

Using a two-column two-level well design will reduce the filtration rate when approaching the filters since the pumps, form the own radial seepage flow to each of the filters during operation. This will create conditions for reducing pressure losses and will reduce the level of groundwater in the well, reducing the cost of produced water, increasing the specific yield of the well and its life compared to a single-column design. Also, the proposed design is more reliable, because if there is a hydraulic connection between the two columns, in case of failure of one filter, working with one pump or two at the same time is possible.

\section{References}

1. A.M. Sheiko, V.V. Ivashechkin, N.V. Kholodinskaya, E.A. Makarova. Vestnik BNTU Analysis of the durability of water wells in Minsk, 27-32 (2006).

2. V. N. Kunin. Moscow: Publishing house Acad. Sciences of the USSR Desert Freshwater Lenses: Research Methods, Resource Assessment and Operation 379 (1963).

3. V.P. Tkachenko. Water well: a.w. 1448002SU, MKI E O3V 3/18. Hydrological expedition of the Ministry of Land Reclamation and Water Resources of the Ukrainian SSR: 29-33 (1988).

4. V. V. Ivashechkin, U.A. Medvedeva, A.N. Kurch. Double-barrel filter water well for the operation of one aquifer 36-41 (2017).

5. Building Design Standards: TKP 45-4.01-30-2009. Water intake facilities (2009). 